\title{
O COMPONENTE ARBÓREO-ARBUSTIVO DE UM REMANESCENTE DE FLORESTA COM ARAUCÁRIA NO MUNICÍPIO DE CURITIBA, PARANÁ
}

\author{
Marlon Selusniaki ${ }^{1}$; Luiz Antonio Acra ${ }^{2}$ \\ ${ }^{1}$ Biólogo, Curador do Herbário (HUCP), PUCPR, Curitiba, PR, Brasil - selusniaki.m@pucpr.br \\ ${ }^{2}$ Biólogo, Depto. de Biologia, PUCPR, Curitiba, PR, Brasil - luiz.acra@pucpr.br \\ Recebido para publicação: 30/09/2009 - Aceito para publicação: 16/12/2009
}

\begin{abstract}
Resumo
Foi feito um levantamento das árvores e arbustos de um remanescente de Floresta Ombrófila Mista, localizado no Bosque da Fazendinha, município de Curitiba, PR, no período de 2004 a 2009, com o objetivo de descrever a composição florística e sua distribuição na área de estudo. Foram registradas 147 espécies, distribuídas em 48 famílias entre angiospermas e gimnospermas. Dentre as Angiospermas, destacaram-se como as mais diversas as famílias Myrtaceae (18), Fabaceae (12), Lauraceae (10) e Asteraceae (nove). As gimnospermas estão representadas apenas por duas famílias, Araucariaceae e Podocarpaceae, cada uma com apenas uma espécie. Foi encontrada expressiva diversidade de espécies apesar desta formação estar situada em área urbana e estar sujeita a pressões de diferentes níveis.

Palavras-chave: Floresta Ombrófila Mista; floresta com araucária; florística.
\end{abstract}

\section{Abstract}

The tree and shrubs compound of a forest remnant with araucaria in Curitiba, Paraná. A floristic survey of trees and shrubs was carried out in the remnants of an Araucaria forest in the 2004-2009 periods, located in the "Bosque da Fazendinha", municipality of Curitiba, Paraná, Brazil, in order to describe the floristic composition and distribution in the study area. A total of 147 species, belonging to 48 families of angiosperms and gymnosperms, were found. Within the Angiosperm group, the most diverse families were Myrtaceae (18), followed by Fabaceae (12), Lauraceae (10) and Asteraceae (9). Gymnosperms were represented by only two families and Araucariaceae and Podocarpaceae byh only one species each. A highly significant diversity in species was observed, even though the formation is located in an urban area and therefore subject to different levels of stress.

Keywords: Mixed Ombrophyllous Forest; araucarian forest; floristic.

\section{INTRODUÇÃO}

A Floresta com Araucária (HUECK, 1972), Floresta Ombrófila Mista (LEITE, 1990) ou ainda Pinheiral (RIZZINI, 1988) é uma das unidades fitoecológicas mais representativas na região Sul do país (IBGE, 1992). O espaço geográfico de distribuição natural do pinheiro-do-paraná está compreendido entre os paralelos $21^{\circ}$ e $30^{\circ} \mathrm{S}$ e meridianos $44^{\circ}$ e $54^{\circ} \mathrm{W}$ (OLIVEIRA, 1948).

Originalmente, a Floresta Ombrófila Mista, no Brasil, cobria aproximadamente $168.000 \mathrm{~km}^{2}$, ocorrendo no estado do Paraná (cerca de $40 \%$ do total), Santa Catarina (31\%), Rio Grande do Sul (25\%) e em manchas esparsas no sul de São Paulo (3\%), internando-se até o sul de Minas Gerais e Rio de Janeiro (1\%) (KLEIN, 1960). No Paraná, era o tipo vegetacional de maior cobertura (MAACK, 1981). Hoje, esse ecossistema restringe-se a pouco mais que $0,7 \%$ da área original em estágios primários ou avançados (MMA, 2002). As indicações mais otimistas registram entre 1 a $2 \%$ de áreas originais cobertas por Floresta com Araucária nos três estados do Sul do país (KOCH; CORRÊA, 2002).

Ao longo do processo histórico de ocupação da região Sul do Brasil, iniciado a partir da década de 1890, assistiu-se a uma rápida eliminação de sua cobertura florestal, tanto para fins de extração como para dar espaço às atividades agropecuárias (MAACK, 1981).

A presença dominante no estrato emergente de Araucaria angustifolia (Bertol.) Kuntze 
caracteriza as chamadas matas escuras (ST.-HILAIRE, 1820) ou matas pretas (REITZ; KLEIN, 1966). A formação com araucária apresenta dois "povoamentos" superpostos: um claro e denso de pinheiros, dominante, consorciado a outro de uma floresta com grande heterogeneidade de espécies, entre elas canelas, louros, pinheiros-bravos e erva-mate (AUBREVILLE, 1949). Embora ela apresente dominância no estrato superior da floresta, os estratos médio e inferior possuem grande diversidade de espécies. Esses estratos são importantes centros de dispersão de um grande número de espécies, principalmente das pertencentes às famílias Myrtaceae e Lauraceae, conforme descrevem Rambo (1951) e Klein (1984).

Informações a respeito da riqueza florística que compõe esse meio natural, em especial no Paraná, são de grande importância para se avaliar as características ecológicas e o dinamismo desse ecossistema. Tais informações podem ser úteis na elaboração e planejamento de ações que objetivam a preservação ou conservação de remanescentes em nível regional, além de fornecer informações básicas para as tomadas de decisão (SANQUETTA, 2000).

As formações de Floresta Ombrófila Mista tiveram sua composição estudada graças ao empenho de vários pesquisadores, como Isernhagen et al. (2001), Longhi (1980), Cervi et al. (1987; 1989), Barddal et al. (2003; 2004), Galvão et al. (1989), Negrelle; Silva (1992), Jarenkow (1985), Carrião (1995), Roseira (1990), Silva; Marconi (1990), Britez et al. (1995), Koehler et al. (1998) e Neto et al. (2002). Entretanto ainda se verifica uma grande necessidade de se desenvolverem estudos abordando esse tema, uma vez que se faz necessário o uso desse conhecimento científico para o desenvolvimento de diretrizes que possam ajudar na conservação da diversidade biológica (TABARELLI; GASCON, 2005).

No município de Curitiba, atualmente, a Floresta com Araucária limita-se a capões isolados em diferentes graus de conservação, localizados na maioria dos casos em parques e bosques municipais, submetidos a diferentes graus de alteração, dado o elevado índice de urbanização da região (BORGO, 2003). Remanescentes de floresta intacta são esparsos e raríssimos, sobretudo em Curitiba e em seus arredores (KOEHLER et al., 1998). Mesmo estando sob proteção do governo, essas áreas continuam sofrendo pressão de natureza humana, o que ocasiona diferentes processos de degradação. Tais áreas, independentemente de sua estrutura florística, representam um refúgio para as espécies da fauna, além de funcionarem como corredores biológicos que proporcionam conectividade aos diversos fragmentos existentes (TABARELLI et al. 2004).

O objetivo do trabalho foi descrever a composição florística arbóreo-arbustiva do Bosque da Fazendinha, bem como essas espécies se distribuem ao longo da área de estudo.

\section{MATERIAL E MÉTODOS}

\section{Localização e caracterização da área de estudo}

O Bosque da Fazendinha localiza-se no Bairro Fazendinha, Rua Carlos Klemtz, porção sudoeste do município de Curitiba, Paraná (2528’34” S e 49¹9’22” W). O bosque foi criado em 1995 e possui uma área total de $72.851 \mathrm{~m}^{2}$, sendo aproximadamente $51.000 \mathrm{~m}^{2}$ de cobertura vegetal, onde se observam as trilhas de lazer (PREFEITURA MUNICIPAL DE CURITIBA, 2007).

A área situa-se a cerca de $920 \mathrm{~m}$ de altitude. Insere-se em região onde predomina o tipo climático $C f b$, subtropical úmido, mesotérmico, segundo a classificação proposta por Koeppen, com temperatura média de $22^{\circ} \mathrm{C}$ no mês mais quente e $12{ }^{\circ} \mathrm{C}$ no mês mais frio. A umidade relativa do ar oscila em torno dos $80 \%$, e a precipitação média é de $1.450 \mathrm{~mm} / \mathrm{ano}$, sendo o mês de janeiro o mais chuvoso, com $170 \mathrm{~mm}$, e agosto o mês mais seco, com $75 \mathrm{~mm}$ (IAPAR, 2007).

O Bosque da Fazendinha está representado pela Floresta Ombrófila Mista Montana, em sua maior extensão, pelas formações aluviais adjacentes à área, oriundas do córrego Formosa e do rio Barigui, e pelas formações campestres da porção oeste do bosque. A vegetação original encontrava-se nos chamados "capões de mata" entremeados por capoeiras e campos naturais, originalmente mais representados nas regiões leste e sul do município. Segundo Maack (1950), a área de Curitiba é assinalada como ocupada pelos campos ao leste e terrenos de culturas efetivas na parte oeste.

\section{Procedimento metodológico}

Para o levantamento florístico, foram realizadas incursões periódicas mensais ao bosque no período de 2004 a 2009, visando coletar todos os exemplares arbustivos e arbóreos encontrados no interior do capão ou em sua extensão de borda. Não foram inclú́das pteridófitas arborescentes e lianas na amostragem. Foram feitas anotações em campo, relacionando a espécie ao seu hábito e posição social na estrutura da comunidade vegetal. 
O material botânico foi coletado, herborizado e posteriormente depositado no Herbário da Universidade Católica (HUCP). As espécies foram identificadas pelo autor sênior com auxílio de bibliografia especializada ou ainda encaminhadas ao Museu Botânico Municipal (MBM).

As angiospermas foram organizadas conforme o sistema APG II (Stevens, 2008). Os nomes das espécies, assim como os autores, foram verificados no Tropicos (2009), e as sinonímias no World Checklist of Selected Plant Families (2009).

\section{RESULTADOS E DISCUSSÃO}

No levantamento realizado, foram encontradas 147 espécies de árvores e arbustos distribuídas em 48 famílias botânicas (Tabela 1). Borgo (2003) realizou um levantamento na mesma área, abordando a florística dos epífitos, e identificou 27 espécies nativas representadas especialmente por Polypodiaceae, Cactaceae e Orchidaceae.

Tabela 1. Relação das famílias e espécies arbóreo-arbustivas observadas no Bosque da Fazendinha ao longo do estudo, seus respectivos tipos biológicos (AR: árvores; $\mathrm{AB}$ : arbustos), origem (N: nativo; E: exótico) e nomes populares.

Table 1. List of families and tree and shrubs species found in the "Bosque da Fazendinha" throughout the study, their biological types (AR: trees; AB: shrubs), origin (N: native; E: exotic) and popular names.

\begin{tabular}{|c|c|c|c|}
\hline $\begin{array}{l}\text { FAMÍLIA (número de espécies) } \\
\text { Espécies }\end{array}$ & Nome Popular & Origem & Hábito \\
\hline \multicolumn{4}{|l|}{ ANACARDIACEAE (3) } \\
\hline Lithraea brasiliensis March. & Aroeira-brava & $\mathrm{N}$ & AR \\
\hline Schinus polygamus (Cav.) Cabrera & Assovieira & $\mathrm{N}$ & AR \\
\hline Schinus terebinthifolius Raddi & Aroeira-vermelha & $\mathrm{N}$ & AR \\
\hline \multicolumn{4}{|l|}{ ANNONACEAE (2) } \\
\hline Rollinia rugulosa Schltdl. & Araticum & $\mathrm{N}$ & AR \\
\hline Xylopia brasiliensis Spreng. & Pandaíba & $\mathrm{N}$ & AR \\
\hline AQUIFOLIACEAE (4) & & & AR \\
\hline Ilex brevicuspis Reissek & Caúna & $\mathrm{N}$ & AR \\
\hline Ilex dumosa Reissek & Congonha, caúna & $\mathrm{N}$ & AR \\
\hline Ilex paraguariensis A. St.-Hil. & Erva-mate & $\mathrm{N}$ & AR \\
\hline Ilex theezans Mart. & Caúna & $\mathrm{N}$ & $\mathrm{AR}$ \\
\hline \multicolumn{4}{|l|}{ ARAUCARIACEAE (1) } \\
\hline Araucaria angustifolia (Bertol.) Kuntze & Pinheiro-do-paraná & $\mathrm{N}$ & AR \\
\hline \multicolumn{4}{|l|}{ ARECACEAE (2) } \\
\hline Rhapis excelsa (Thunb.) A. Henry ex Rehder & Palmeira-ráfia & E & AR \\
\hline Syagrus romanzoffiana (Cham.) Glassman & Jerivá & $\mathrm{N}$ & AR \\
\hline \multicolumn{4}{|l|}{ ASTERACEAE (9) } \\
\hline Baccharis dracunculifolia DC. & Vassourinha & $\mathrm{N}$ & $\mathrm{AB}$ \\
\hline Baccharis semiserrata DC. & Vassourinha & $\mathrm{N}$ & $\mathrm{AB}$ \\
\hline Dasyphyllum tomentosum (Spreng.) Cabrera & Açucará, guaiapá & $\mathrm{N}$ & $\mathrm{AR}$ \\
\hline Eupatorum laevigatum Baker & Cambará-falso & $\mathrm{N}$ & $\mathrm{AB}$ \\
\hline Gochnatia polymorpha (Less.) Cabrera & Cambará & $\mathrm{N}$ & AR \\
\hline Pterocaulon balansae Chodat & Verbasco & $\mathrm{N}$ & $\mathrm{AB}$ \\
\hline Piptocarpha angustifolia Dusén ex Malme & Vassourão-branco & $\mathrm{N}$ & AR \\
\hline Piptocarpha axillaris (Less.) Baker & Vassourão & $\mathrm{N}$ & AR \\
\hline Vernonia discolor (Spreng.) Less. & Vassourão-preto & $\mathrm{N}$ & AR \\
\hline \multicolumn{4}{|l|}{ BIGNONIACEAE (2) } \\
\hline Jacaranda puberula Cham. & Caroba, jacarandá & $\mathrm{N}$ & AR \\
\hline Tabebuia alba (Cham.) Sandw. & Ipê-da-serra & $\mathrm{N}$ & $\mathrm{AR}$ \\
\hline \multicolumn{4}{|l|}{ CANELLACEAE (1) } \\
\hline $\begin{array}{l}\text { Capsicodendron dinisii (Schwacke) Occhioni } \\
\text { CANNABACEAE (1) }\end{array}$ & Pimenteira & $\mathrm{N}$ & AR \\
\hline
\end{tabular}

FLORESTA, Curitiba, PR, v. 40, n. 3, p. 593-602, jul./set. 2010. 


\begin{tabular}{|c|c|c|c|}
\hline Celtis iguanaea (Jacq.) Sarg. & Taleira & $\mathrm{N}$ & AR \\
\hline \multicolumn{4}{|l|}{ CARDIOPTERIDACEAE (1) } \\
\hline Citronella gongonha (Mart.) Howard & Congonha & $\mathrm{N}$ & AR \\
\hline \multicolumn{4}{|l|}{ CELASTRACEAE (1) } \\
\hline Maytenus ilicifolia Reissek & Espinheira-santa & $\mathrm{N}$ & AR \\
\hline \multicolumn{4}{|l|}{ CLETHRACEAE (1) } \\
\hline Clethra scabra Pers. & Caujuja, caijuja & $\mathrm{N}$ & AR \\
\hline \multicolumn{4}{|l|}{ CUNONIACEAE (1) } \\
\hline Lamanonia speciosa (Camb.) L.B. Smith & Guaraperê & $\mathrm{N}$ & AR \\
\hline \multicolumn{4}{|l|}{ ELAEOCARPACEAE (1) } \\
\hline Sloanea monosperma Vell. & Sapopema & $\mathrm{N}$ & AR \\
\hline \multicolumn{4}{|l|}{ ERYTHROXYLACEAE (1) } \\
\hline Erythroxylum deciduum A. St.-Hil. & Concon, cocão & $\mathrm{N}$ & AR \\
\hline \multicolumn{4}{|l|}{ EUPHORBIACEAE (3) } \\
\hline Sapium glandulatum (Vell.) Pax. & Tapiá, tapurú & $\mathrm{N}$ & AR \\
\hline Sebastiania brasiliensis Spreng. & Branquinho, tajuva & $\mathrm{N}$ & AR \\
\hline Sebastiania commersoniana (Baill.) Smith et Downs & Branquilho & $\mathrm{N}$ & AR \\
\hline \multicolumn{4}{|l|}{ FABACEAE (12) } \\
\hline Acacia recurva Benth. & Acácia & $\mathrm{N}$ & $A B$ \\
\hline Bauhinia forficata Link & Pata-de-vaca & $\mathrm{E}$ & AR \\
\hline Calliandra brevipes Benth. & Sarandi & $\mathrm{E}$ & $\mathrm{AB}$ \\
\hline Collaea speciosa Loisel & Coleia & $\mathrm{N}$ & $\mathrm{AB}$ \\
\hline Dalbergia brasiliensis Vog. & Marmeleiro, cateretê & $\mathrm{N}$ & AR \\
\hline Dalbergia frutescens (Vell.) Britton & Farinha-seca, cateretê & $\mathrm{N}$ & AR \\
\hline Lonchocarpus subglaucescens Mart. ex Benth. & Rabo-de-bugiu & $\mathrm{N}$ & AR \\
\hline Machaerium paraguariense Hassl. & Sapuvuçu & $\mathrm{N}$ & AR \\
\hline Machaerium stipitatum (DC.) Vog. & Sapuva & $\mathrm{N}$ & AR \\
\hline Mimosa dolens Vell. var. acerba (Benth.) Barneby & Mimosa & $\mathrm{N}$ & $\mathrm{AB}$ \\
\hline Mimosa scabrella Benth. & Bracatinga & $\mathrm{N}$ & AR \\
\hline Senna macranthera (DC. ex Collad.) H.S. Irwin et Barneby & Chuva-de-ouro & $\mathrm{E}$ & AR \\
\hline \multicolumn{4}{|l|}{ LAMIACEAE (2) } \\
\hline Salvia splendens Sellow ex Wied-Neuw. & Alegria-dos-jardins & $\mathrm{E}$ & $A B$ \\
\hline Vitex megapotamica (Spreng.) Moldenke & Tarumã & $\mathrm{N}$ & AR \\
\hline \multicolumn{4}{|l|}{ LAURACEAE (10) } \\
\hline Сіппатотит атоепит (Nees) Kosterm. & Canela-fedida, pau-d'alho & $\mathrm{N}$ & AR \\
\hline Cinnamoтит sellowianum (Nees et Mart. ex Nees) Kosterm. & Canela & $\mathrm{N}$ & AR \\
\hline Cryptocarya aschersoniana Mez & Canela-fogo & $\mathrm{N}$ & AR \\
\hline Nectandra lanceolata Nees & Canela-amarela & $\mathrm{N}$ & AR \\
\hline Ocotea bicolor Vattimo & Canela & $\mathrm{N}$ & AR \\
\hline Ocotea nutans (Ness) Mez & Canela-vermelha & $\mathrm{N}$ & AR \\
\hline Ocotea porosa (Nees et Mart.) Barroso & Imbuia & $\mathrm{N}$ & AR \\
\hline Ocotea puberula (Rich.) Nees & Canela-guaicá & $\mathrm{N}$ & AR \\
\hline Ocotea pulchella (Ness) Mez & Canela-lageana & $\mathrm{N}$ & AR \\
\hline Persea americana $\mathrm{L}$. & Abacateiro & $\mathrm{E}$ & AR \\
\hline \multicolumn{4}{|l|}{ LOGANIACEAE (1) } \\
\hline Strychnos brasiliensis (Spreng.) Mart. & Quina, esporão & $\mathrm{N}$ & $A B$ \\
\hline \multicolumn{4}{|l|}{ MALVACEAE (3) } \\
\hline Chorisia speciosa A. St.-Hil. & Paineira & $\mathrm{E}$ & AR \\
\hline Luehea divaricata Mart. & Açoita-cavalo, ibatingui & $\mathrm{N}$ & AR \\
\hline Malvaviscus arboreus Cav. & Malvavisco & $\mathrm{E}$ & $\mathrm{AB}$ \\
\hline \multicolumn{4}{|l|}{ MELASTOMATACEAE (6) } \\
\hline Leandra erostrata (DC.) Cogn. & Pixirica-do-campo & $\mathrm{N}$ & $\mathrm{AB}$ \\
\hline Leandra polystachya (Naud.) Cogn. & Pixirica-do-campo & $\mathrm{N}$ & $A B$ \\
\hline Leandra sylvestris DC. & Pixirica & $\mathrm{N}$ & $\mathrm{AB}$ \\
\hline
\end{tabular}




\begin{tabular}{|c|c|c|c|}
\hline Miconia hyemalis A. St.-Hil. et Naudin ex Naudin & Jacatirão & $\mathrm{N}$ & $\mathrm{AB}$ \\
\hline Miconia petropolitana Cogn. & Pixirica & $\mathrm{N}$ & $\mathrm{AB}$ \\
\hline Tibouchina trichopoda Baill. & Manacá-do-campo & $\mathrm{N}$ & $\mathrm{AB}$ \\
\hline \multicolumn{4}{|l|}{ MELIACEAE (2) } \\
\hline Cedrela fissilis Vell. & Cedro-rosa, acaju-catinga & $\mathrm{N}$ & AR \\
\hline Trichilia elegans Juss. & Catiguá & $\mathrm{N}$ & AR \\
\hline \multicolumn{4}{|l|}{ MONIMIACEAE (1) } \\
\hline Mollinedia clavigera Tulasne & Capixim & $\mathrm{N}$ & $\mathrm{AB}$ \\
\hline \multicolumn{4}{|l|}{ MORACEAE (3) } \\
\hline Morus nigra $\mathrm{L}$. & Amoreira & $\mathrm{E}$ & $\mathrm{AR}$ \\
\hline Sorocea bonplandii (Baill.) Burger, Lanj et Boer & Carapicica & $\mathrm{N}$ & AR \\
\hline \multicolumn{4}{|l|}{ MUSACEAE (1) } \\
\hline Musa x paradisiaca $\mathrm{L}$. & Bananeira & $\mathrm{E}$ & AR \\
\hline \multicolumn{4}{|l|}{ MYRSINACEAE (3) } \\
\hline Myrsine ferruginea (Ruiz et Pav.) Spreng. & Capororoquinha & $\mathrm{N}$ & AR \\
\hline Myrsine gardneriana A. DC. & Capororoca & $\mathrm{N}$ & AR \\
\hline Myrsine umbellata Mart. & Capororocão & $\mathrm{N}$ & AR \\
\hline \multicolumn{4}{|l|}{ MYRTACEAE (18) } \\
\hline Campomanesia adamantium (Camb.) Berg & Guaviroba-do-campo & $\mathrm{N}$ & $\mathrm{AB}$ \\
\hline Campomanesia guazumifolia (Camb.) Berg & Guaviroba & $\mathrm{N}$ & $\mathrm{AR}$ \\
\hline Campomanesia xanthocarpa Berg & Guabiroba & $\mathrm{N}$ & $\mathrm{AR}$ \\
\hline Curitiba prismatica (Legr.) Salywon et Land. & Guamirim-miúdo & $\mathrm{N}$ & AR \\
\hline Eugenia neoverrucosa Sobral & Pitanga-do-mato & $\mathrm{N}$ & AR \\
\hline Eugenia pluriflora DC. & Jaboticaba-do-mato & $\mathrm{N}$ & $\mathrm{AB}$ \\
\hline Eugenia uniflora $\mathrm{L}$. & Pitanga & $\mathrm{N}$ & $\mathrm{AR}$ \\
\hline Gomidesia palustris (DC.) Legr.* & Guamirim & $\mathrm{N}$ & AR \\
\hline Myrceugenia euosma (Berg) Legr. & Cambuizinho & $\mathrm{N}$ & $\mathrm{AB}$ \\
\hline Myrceugenia miersiana (Gardner) Legr. et Kausel & Cambuí & $\mathrm{N}$ & $\mathrm{AB}$ \\
\hline Myrcia arborescens Berg & Guamirim & $\mathrm{N}$ & AR \\
\hline Myrcia guianensis (Aubl.) DC.** & Guamirim-branco & $\mathrm{N}$ & $\mathrm{AB}$ \\
\hline Myrcia hatschbachii Legr. & Caaingá, guamirim-ferro & $\mathrm{N}$ & $\mathrm{AR}$ \\
\hline Myrcia laruotteana Camb. & Guamirim-vermelho & $\mathrm{N}$ & $\mathrm{AR}$ \\
\hline Myrcia rostrata DC. & Cambuizinho & $\mathrm{N}$ & AR \\
\hline Myrciaria tenella (DC.) Berg & Cambuí & $\mathrm{N}$ & $\mathrm{AB}$ \\
\hline Psidium australe Camb. & Araçá-do-campo & $\mathrm{N}$ & $\mathrm{AB}$ \\
\hline Psidium cattleianum Sabine & Araçá & $\mathrm{N}$ & AR \\
\hline \multicolumn{4}{|l|}{ OLEACEAE (1) } \\
\hline Ligustrum lucidum W.T. Aiton & Alfeneiro & $\mathrm{E}$ & $\mathrm{AR}$ \\
\hline \multicolumn{4}{|l|}{ OPILIACEAE (1) } \\
\hline Agonandra englerii Hoehne & Tinge-cuia, pau-tatu & $\mathrm{N}$ & AR \\
\hline \multicolumn{4}{|l|}{ PIPERACEAE (2) } \\
\hline Piper gaudichaudianum Kunth & Pimentinha & $\mathrm{N}$ & $\mathrm{AB}$ \\
\hline Piper mikanianum (Kunth) Steud. & Pimenta-do-mato & $\mathrm{N}$ & $\mathrm{AB}$ \\
\hline \multicolumn{4}{|l|}{ PITTOSPORACEAE (1) } \\
\hline Pittosporum undulatum Vent. & Pau-incenso, pitósporo & $\mathrm{E}$ & $\mathrm{AR}$ \\
\hline \multicolumn{4}{|l|}{ PODOCARPACEAE (1) } \\
\hline Podocarpus lambertii Klotz. ex Endl. & Pinheiro-bravo & $\mathrm{N}$ & $\mathrm{AR}$ \\
\hline \multicolumn{4}{|l|}{ PROTEACEAE (1) } \\
\hline Roupala brasiliensis Klotz. & Carvalho-brasileiro & $\mathrm{N}$ & $\mathrm{AR}$ \\
\hline \multicolumn{4}{|l|}{ RHAMNACEAE (2) } \\
\hline Hovenia dulcis Thunb. & Uva-do-japão & $\mathrm{E}$ & $\mathrm{AR}$ \\
\hline Rhamnus sphaerosperma $\mathrm{Sw}$. & Tarumaí, cangica & $\mathrm{N}$ & $\mathrm{AB}$ \\
\hline \multicolumn{4}{|l|}{ ROSACEAE (4) } \\
\hline Eriobotrya japonica (Thunb.) Lindl. & Néspera & $\mathrm{E}$ & $\mathrm{AR}$ \\
\hline
\end{tabular}

FLORESTA, Curitiba, PR, v. 40, n. 3, p. 593-602, jul./set. 2010. 


\begin{tabular}{|c|c|c|c|}
\hline Prunus brasiliensis Mart.*** & Pessegueiro-do-mato & $\mathrm{N}$ & $\mathrm{AR}$ \\
\hline Prunus sellowii Koehne*** & Pessegueiro-bravo & $\mathrm{N}$ & $\mathrm{AR}$ \\
\hline Rubus rosaefolius J. Sm. & Amora-do-mato & $\mathrm{N}$ & $\mathrm{AB}$ \\
\hline \multicolumn{4}{|l|}{ RUBIACEAE (7) } \\
\hline Coffea arabica $\mathrm{L}$. & Café & $\mathrm{E}$ & $\mathrm{AB}$ \\
\hline Guettarda uruguensis Cham. et Schltdl. & Veludo & $\mathrm{N}$ & $\mathrm{AB}$ \\
\hline Psychotria suturella Muell. Arg. & Grandiúva & $\mathrm{N}$ & $\mathrm{AB}$ \\
\hline Psychotria vellosiana Benth. & Grandiúva-d'anta & $\mathrm{N}$ & AR \\
\hline Randia ferox (Cham. et Schldtl.) A. DC. & Limoeiro-do-mato & $\mathrm{N}$ & AR \\
\hline Rudgea jasminioides (Cham.) Muell. Arg. & Jasmin-do-mato & $\mathrm{N}$ & $\mathrm{AB}$ \\
\hline Rudgea parquioides (Cham.) Muell. Arg. & Pimentinha & $\mathrm{N}$ & $\mathrm{AB}$ \\
\hline \multicolumn{4}{|l|}{ RUTACEAE (3) } \\
\hline Citrus aurantium $\mathrm{L}$. & Laranjeira & $\mathrm{E}$ & AR \\
\hline Zanthoxylum kleinii (Cowan) Waterman & Tembetari, juva & $\mathrm{N}$ & AR \\
\hline Zanthoxylum rhoifolium Lam. & Juvevê & $\mathrm{N}$ & AR \\
\hline \multicolumn{4}{|l|}{ SALICACEAE (7) } \\
\hline Banara tomentosa Clos & Cabroé-mirim & $\mathrm{N}$ & AR \\
\hline Casearia decandra Jacq. & Guaçatunga-preta & $\mathrm{N}$ & AR \\
\hline Casearia lasiophylla Eichler & Cambroé & $\mathrm{N}$ & AR \\
\hline Casearia obliqua Spreng. & Guaçatunga-vermelha & $\mathrm{N}$ & AR \\
\hline Casearia sylvestris $\mathrm{Sw}$. & Guaçatunga & $\mathrm{N}$ & AR \\
\hline Xylosma ciliatifolium (Clos) Eichler & Sucará & $\mathrm{N}$ & AR \\
\hline Xylosma pseudosalzmanii Sleumer & Sucará & $\mathrm{N}$ & AR \\
\hline \multicolumn{4}{|l|}{ SAPINDACEAE (3) } \\
\hline Allophylus edulis (A. St.-Hil.) Radlk. & Vacum & $\mathrm{N}$ & AR \\
\hline Cupania vernalis $\mathrm{Camb}$. & Cuvatá, cuvatã & $\mathrm{N}$ & AR \\
\hline Matayba elaeagnoides Radlk. & Camboatá & $\mathrm{N}$ & AR \\
\hline \multicolumn{4}{|l|}{ SIMAROUBACEAE (2) } \\
\hline Picramnia parviflora Engler & Pau-amargo & $\mathrm{N}$ & $\mathrm{AR}$ \\
\hline Picrasma crenata Engl. in Engl. et Prantl & Pau-amargo, queina & $\mathrm{N}$ & AR \\
\hline \multicolumn{4}{|l|}{ SOLANACEAE (8) } \\
\hline Acnistus arborescens (L.) Schldtl. & Esporão-de-galo & $\mathrm{N}$ & AR \\
\hline Aureliana fasciculata (Vell.) Sendtn. & Belonha-miúda & $\mathrm{N}$ & $\mathrm{AB}$ \\
\hline Cestrum amictum Schltdl. & Coerana & $\mathrm{N}$ & $\mathrm{AB}$ \\
\hline Solanum compressum Smith et Downs & Coerana & $\mathrm{N}$ & AR \\
\hline Solanum granuloso-leprosum Dunal & Canema & $\mathrm{N}$ & AR \\
\hline Solanum paranense Dusén & Canema-miúda & $\mathrm{N}$ & $\mathrm{AB}$ \\
\hline Solanum pseudoquina A. St.-Hil. & Joá-manso & $\mathrm{N}$ & AR \\
\hline Solanum sanctae-catharinae Dunal & Cuvitinga & $\mathrm{N}$ & $\mathrm{AR}$ \\
\hline \multicolumn{4}{|l|}{ STYRACACEAE (1) } \\
\hline Styrax leprosus Hook. et Arn. & Pau-de-remo, jaguatinga & $\mathrm{N}$ & AR \\
\hline \multicolumn{4}{|l|}{ SYMPLOCACEAE (2) } \\
\hline Symplocos tetrandra Mart. & Sete-sangrias & $\mathrm{N}$ & AR \\
\hline Symplocos uniflora (Pohl) Benth. & Pau-de-cangalha & $\mathrm{N}$ & AR \\
\hline \multicolumn{4}{|l|}{ THYMELAEACEAE (1) } \\
\hline Daphnopsis racemosa Griseb. & Embira-branca, ibiratinga & $\mathrm{N}$ & $\mathrm{AB}$ \\
\hline \multicolumn{4}{|l|}{ VERBENACEAE (4) } \\
\hline Duranta vestita Cham. & Baga-de-pomba, grão-de-galo & $\mathrm{N}$ & AR \\
\hline Lantana brasiliensis Link & Camará-tinga, camará & $\mathrm{N}$ & $\mathrm{AB}$ \\
\hline Lantana fucata Lindl. & Camará & $\mathrm{N}$ & $\mathrm{AB}$ \\
\hline Stachytarpheta cayennensis (Rich.) Vahl & Gervão & $\mathrm{N}$ & $\mathrm{AB}$ \\
\hline \multicolumn{4}{|l|}{ WINTERACEAE (1) } \\
\hline Drimys brasiliensis Miers & Cataia & $\mathrm{N}$ & $\mathrm{AR}$ \\
\hline
\end{tabular}

*Citada por Marchiori e Sobral (1997) como Gomidesia palustris (DC.) Kausel. **Conforme Sobral et al. (2006), a espécie Myrcia guianensis vinha sendo erroneamente identificada como Myrcia obtecta, no Sul do país. ***Citadas por Sobral et al. (2006) como Prunus myrtifolia. 
As famílias com maior riqueza específica foram Myrtaceae (18 espécies), Fabaceae (12), Lauraceae (10), Asteraceae (9), Solanaceae (8), Salicaceae e Rubiaceae (7) e Melastomataceae (6), o que compõem mais de $50 \%$ da diversidade florística do parque.

Esses resultados se mostram coerentes com os estudos realizados por pesquisadores em diferentes áreas de Floresta Ombrófila Mista, no Brasil, a citar Jarenkow (1985), Calegari (1999), Barddal e colaboradores (2003; 2004), Longhi (1997) e Koehler e colaboradores (1998). No que diz respeito à floresta, o dossel, com cerca de 20 $\mathrm{m}$, é dominado por alguns pinheiros e algumas canelas, como a Ocotea porosa, O. puberula, Nectandra lanceolata, Jacaranda puberula e Cedrella fissilis. O estrato médio possui em torno de $14 \mathrm{~m}$ e se caracteriza principalmente por apresentar indivíduos das famílias Asteraceae, Myrtaceae, Proteaceae, Lauraceae e Podocarpaceae. Eventualmente, espécies oportunistas que aparecem quando do surgimento de uma clareira, como Passifloraceae, Bignoniaceae, Sapindaceae e outras trepadeiras, são observadas na transição do estrato médio ao superior. Já no estrato inferior, cerca de $10 \mathrm{~m}$, ocorre uma grande abundância e densidade de indivíduos das famílias Monimiaceae, Myrsinaceae, Sapindaceae e Salicaceae. Indivíduos de menor porte que compõem o subbosque da floresta estão representados em sua maioria por Strychnos brasiliensis, Miconia spp., Eugenia pluriflora, Piper spp. e, em grande abundância, Mollinedia clavigera.

Corroborando os trabalhos anteriores, nota-se que a família Myrtaceae possui elevada ocorrência específica e representatividade em diversos tipos florestais. A diversidade encontrada de espécies arbóreas e arbustos dessa família é expressiva, como também observam Rambo (1951), Klein (1984) e Jarenkow (1985). Os mesmos autores ainda afirmam que essas comunidades vegetais constituem um importante centro de dispersão dessa família, na qual se observam desde árvores de grande porte até árvores menores e arbustos, que habitam o sub-bosque da vegetação.

Segundo Schäffer; Prochnow (2002), na Floresta com Araucária, o pinheiro-do-paraná representa, em muitos casos, mais de $40 \%$ dos indivíduos arbóreos em condições naturais de conservação. No entanto, isso não pôde ser observado na área de estudo. Poucos indivíduos de pinheiro-do-paraná de grande porte, outrora mais abundantes, foram encontrados. Nota-se também uma redução no número desses indivíduos se regenerando no sub-bosque, o que nos mostra que indivíduos como os de $27 \mathrm{~m}$ de altura encontrados são remanescentes de épocas em que a floresta era melhor estruturada, sem demasiada interferência humana.

Nas porções de influência fluvial, mais próximas da borda oriental do capão, encontraram-se muitos indivíduos de Sebastiania commersoniana, Allophylus edulis e Schinus terebinthifolius.

Nas formações campestres, encontraram-se muitas espécies que ainda caracterizam o ecossistema, apesar de seu estado de degradação devido ao intenso pisoteio e às queimadas. Indivíduos de Maytenus ilicifolia, Pterocaulon balansae, Leandra polystachya, L. erostrata, Mimosa dolens, Tibouchina trichopoda, Psidium australe e Campomanesia adamantium somente foram encontrados nessa formação típica. Outras espécies de gramíneas, asteráceas e algumas leguminosas, não citadas na listagem, também foram encontradas nessa tipologia.

A diversidade florística do Bosque da Fazendinha pode ser considerada satisfatória para a formação quando comparada aos levantamentos realizados na região de Curitiba, como é o caso de Kozera et al. (2006), Cervi et al. (1987), Dombrowski; Kuniyoshi (1967) e Roseira (1990). Em todos esses trabalhos, a diversidade foi menor do que aquela obtida para o Bosque da Fazendinha, exceto o de Kozera et al. (2006). Em parte, isso traduz a natureza do levantamento e a forma de seu desenrolar, uma vez que nesta amostragem foram listados somente os indivíduos arbóreos e arbustivos, não se levando em conta as espécies herbáceas, as lianas e as Pteridophyta, o que confere outro aspecto aos resultados. Outro fator que influenciaria na florística seria a ocorrência de formações mais diversas do Parque Barigui, especialmente as de influência do regime hídrico do rio de mesmo nome. Kozera et al. (2006) encontraram 141 espécies arbóreas e 67 arbustivas no Parque Barigui. Nota-se uma maior variedade de Ilex, Maytenus, ou mesmo a ocorrência de gêneros não vistos no bosque, como é o caso de Ficus spp., Cabralea ou mesmo Tetrapanax, típicos das formações com araucária.

Borgo (2003) classificou a vegetação do Bosque da Fazendinha em seu estudo como "fragmento em estádio intermediário a avançado de regeneração com atividade humana evidente, mas sem causar alterações drásticas no componente florístico, ou, ainda, área com histórico de alterações evidentes, mas que atualmente não afetam a estrutura do fragmento". Contudo, notou-se no estudo a influência direta da pressão antrópica sobre a diversidade local, além do aparecimento de espécies exóticas ou ainda a inexistência de indivíduos para recompor a estrutura avançada da área. Algumas espécies listadas não são observadas naturalmente nesses ambientes, como é o caso de Chorisia speciosa, Senna macranthera ou ainda Bauhinia forficata. Salvia e Malvaviscus são outros exemplos encontrados no local, comumente utilizadas como ornamentais nas áreas urbanas. Indivíduos de alfeneiro, pau-incenso, amoreira, uva-do-japão, néspera ou mesmo café também foram encontrados no interior do bosque, provavelmente trazidos pela avifauna local. 
Com relação ainda à interferência das espécies exóticas na estrutura da vegetação, o alfeneiro (Ligustrum lucidum), espécie arbórea exótica, destacou-se como a mais importante na amostragem de Roseira (1990), evidenciando forte interferência no ambiente, através da descaracterização da estrutura da vegetação original.

Evidenciou-se também que a estrutura da floresta, em especial a dos estratos médio e inferior, está sofrendo importantes modificações, pela abertura de trilhas secundárias. De acordo com Borgo (2003), o aparecimento de clareiras gera alterações diretas na intensidade de luz, umidade, ventos e microclimas. Ao longo da amostragem, foi observada a queda natural de algumas canelas e pimenteiras, ocasionando quedas de indivíduos menores do estrato inferior e do sub-bosque e o posterior estabelecimento de lianas e outras espécies heliófitas.

\section{CONCLUSÕES E RECOMENDAÇÕES}

A vegetação nativa do Bosque da Fazendinha possui grande diversidade de espécies, mesmo encontrando-se em área urbana e antropizada. A elevada riqueza encontrada justifica a contínua conservação dessa área. Fica evidente que a intensa visitação ao bosque, bem como o surgimento de trilhas secundárias e deposição de lixo, o tornam gradativamente vulnerável, atuando diretamente nos processos de dinâmica do capão. Devido às condições de impacto a que essas áreas estão sujeitas, faz-se necessária uma significativa reestruturação do uso dos parques municipais, buscando-se conscientizar a população da importância da manutenção e existência dessas áreas em meio urbano.

\section{AGRADECIMENTOS}

Ao Prof. Dr. Rodrigo Kersten, pelo grande apoio, ajuda e incentivo nas etapas da pesquisa. Ao Prof. Luiz A. Acra, pela realização do trabalho, grande ajuda e companheirismo ao longo dos anos. Aos amigos Ricardo Moledo e Kalliu Zimmer, pela amizade e ajuda.

\section{REFERÊNCIAS}

Aubreville, A. A Floresta de Pinho do Brasil. Anuário Brasileiro de Economia Florestal, Rio de Janeiro, n. 2, p. 21-36, 1949.

BARDDAL, M. L.; PROTIL, C. Z.; SOUZA, S. G. A. de Fitossociologia do componente arbóreo de um trecho da planície do rio Iraízinho, Piraquara, Paraná, Brasil. Estudos de Biologia, Curitiba, v. 25, n. 53, p. 17-28, out/dez, 2003.

BARDDAL, M. L.; RODERJAN, C. V.; GALVÃO, F. CURCIO, G. R. Fitossociologia do Sub-bosque de uma Floresta Ombrófila Mista Aluvial, no município de Araucária, PR. Ciência Florestal, Santa Maria, v. 14, n. 1, p. 35-45, 2004.

BORGO, M. Epífitos vasculares em fragmentos de Floresta Ombrófila Mista, Curitiba, Paraná, Brasil. Dissertação (Mestrado em Botânica) - Setor de Ciências Biológicas, Universidade Federal do Paraná, Curitiba, 2003.

BRASIL. Ministério do Meio Ambiente. Proposta do grupo de trabalho preservação e recuperação da Floresta Ombrófila Mista no estado de Santa Catarina. Brasília, DF, 2002, p. 77. Portaria Ministerial 49 de 06 de fevereiro de 2002.

BRITEZ, R. M.; SILVA, S. M.; SOUZA, W. S.; MOTTA, J. T. W. Levantamento florístico em floresta Ombrófila Mista, São Mateus do Sul, PR, Brasil. Arquivos de Biologia e Tecnologia, Curitiba, v. 38, p. 1147-1161, 1995.

CALEGARI, J. Tamanho ótimo da unidade amostral para estudo da regeneração natural de uma Floresta Ombrófila Mista. Dissertação (Mestrado em Engenharia Florestal) - Universidade Federal de Santa Maria, Santa Maria, 1999.

CARRIÃO, D. J. S. Estudo Fitossociológico do estrato arbóreo do Parque Barigui, Curitiba, PR. Dados preliminares. CONGRESSO NACIONAL DE BOTÂNICA, 46., 1995, Ribeirão Preto). Resumos... Ribeirão Preto, p. 99, 1995. 
CERVI, A. C.; PACIORNIK, E. F.; VIEIRA, R. F.; MARQUES, L. C. Espécies vegetais de um remanescente de Floresta de araucária (Curitiba, Brasil): Estudo preliminar I. Acta Biológica Paranaense, Curitiba, v. 18, n. 4, p. 73-114, 1989.

CERVI, A. C.; SCHIMMELPFENG, L. C. T.; PASSOS, M. Levantamento do estrato arbóreo do capão de educação física da Universidade Federal do Paraná, Curitiba - PR - Brasil. Estudos de Biologia, Curitiba, n. 17, p. 49-61, 1987.

CURITIBA. Prefeitura Municipal. Disponível em: <www.curitiba.pr.gov.br/Servicos/MeioAmbiente/ areas_verdes/parques_bosques/bosque_fazendinha.htm>. Acesso em: 22/3/2007.

DOMBROWSKI, L. T. D.; KUNIYOSHI, Y. S. A vegetação do “Capão da Imbuia”. Araucariana. Série Botânica, Curitiba, n. 1, p. 1-18, 1967.

GALVÃO, F.; KUNIYOSHI, Y. S.; RODERJAN, C. V. Levantamento fitossociológico das principais associações arbóreas da floresta nacional de Irati - PR. Floresta, Curitiba, v. 19, n. 1 e 2, p. 30-49, 1989.

HATSCHBACH, G.; KLEIN, R. M. Fitofisionomia e notas sobre a vegetação para acompanhar a planta fitogeográfica do município de Curitiba e arredores (Paraná). Boletim da Universidade do Paraná, Geografia Física. Curitiba, UFPR, n. 4, 1962.

HUECK, K. As florestas da América do Sul. São Paulo: Universidade de São Paulo, 1972.

INSTITUTO AGRONÔMICO DO PARANÁ (IAPAR). Cartas climáticas do estado. Instituto Agronômico do Paraná. Disponível em: <http://www.iapar.br/sma/cartas_climaticas /cartas_climaticas.htm>. Acesso em: 15/6/2007.

INSTITUTO BRASILEIRO DE GEOGRAFIA E ESTATÍSTICA (IBGE). Manual Técnico da vegetação brasileira, Rio de Janeiro, p. 92, 1992.

ISERNHAGEN, I.; SILVA, S. M.; GALVÃO, F. A fitossociologia florestal no Paraná: listagem bibliográfica comentada. 2001. Disponível em: <http://www.ipef.br/servicos/teses>. Acesso em: 13/9/2009.

JARENKOW, J. A. Composição florística e estrutura da mata com araucária na Estação Ecológica de Aracuri, Esmeralda, Rio Grande do Sul. Dissertação (Mestrado em Botânica) - Universidade Federal do Rio Grande do Sul, Porto Alegre, 1985.

KLEIN, R. M. O aspecto dinâmico do pinheiro brasileiro. Sellowia, Itajaí, n. 12, p. 17-44, 1960.

KLEIN, R. M. Importância sociológica das mirtáceas nas florestas rio-grandenses. In: CONGRESSO NACIONAL DE BOTÂNICA, 34, 1984, Manaus. Anais... Manaus: Sociedade Botânica do Brasil, p. 367-375, 1984.

KOCH, Z.; CORRÊA, M. S. Araucária: a floresta do Brasil meridional. Curitiba: Olhar Brasileiro, 2002.

KOEHLER, A. B.; PÉLliCO NETTO, S.; SANQUETA, C. R. Análise da estrutura de uma Floresta Ombrófila Mista semi-devastada, Fazenda Gralha Azul, Região Metropolitana de Curitiba, com implicações ao manejo. Acadêmica, Curitiba, v. 9, n. 1, p. 37-60, 1998.

KOEPPEN, W. Climatologia: com um estúdio de los climas de la tierra. México, 1948.

KOZERA, C.; DITRIICH, V. A. de O.; SILVA, S. M. Composição florística da Floresta Ombrófila Mista Montana do Parque Municipal do Barigui, Curitiba, PR. Floresta, Curitiba, v. 36, n. 1, jan./abr. 2006.

LEITE, P. F. As diferentes unidades fitoecológicas da região sul do Brasil - proposta de classificação. Dissertação (Mestrado em Engenharia Florestal) - Setor de Ciências Agrárias, Universidade Federal do Paraná, Curitiba. 1990.

LONGHI, S. J. A estrutura de uma floresta natural de Araucaria angustifolia (Bertol.) Kuntze no sul do Brasil. Dissertação (Mestrado em Botânica) - Setor de Ciências Biológicas, Universidade Federal do Paraná, Curitiba. 1980. 
LONGHI, S. J. Agrupamento e análise fitossociológica em comunidades florestais na sub-bacia do rio Passo Fundo, RS. Tese (Doutorado em Engenharia Florestal) - Setor de Ciências Agrárias, Universidade Federal do Paraná, Curitiba, 1997.

MAACK, R. Mapa fitogeográfico do estado do Paraná. 1 mapa: color.; 80 x 115 cm; escala 1:750.000. Curitiba: Serviço de Geologia e Petrografia do Instituto de Biologia e Pesquisas Tecnológicas da Secretaria de Agricultura, Indústria e Comércio e Instituto Nacional do Pinho, 1950.

MAACK, R. Geografia Física do estado do Paraná. Curitiba: CODEPAR, p. 350, 1981.

MARCHIORI, J. N. C.; SOBRAL, M. Dendrologia das angiospermas: Myrtales. Santa Maria: UFSM, 1997.

NEGRELLE, R. A. B.; SILVA, F. C. da Fitossociologia de um trecho de Floresta com Araucaria angustifolia (Bertol.) Kuntze no município de Caçador - SC. Boletim de Pesquisa Florestal, Colombo, n. 24/25, p. 37-54, jan/dez, 1992.

OLIVEIRA, B. As regiões de ocorrência da Araucária. Anuário Brasileiro de Economia Floresta, Rio de janeiro, n. 1, p.185-199, 1948.

RAMBO, B. O elemento andino pinhal rio-grandense. Anais Botânicos do Herbário Barbosa Rodrigues, Itajaí, v. 3, n. 3, p. 3-39, 1951.

REITZ, R.; KLEIN, R. M. Flora Ilustrada Catarinense. Araucariáceas. Itajaí: Herbário Barbosa Rodrigues, 1966.

RIZZINI, C. T. Ecossistemas Brasileiros. [S.I.]: Index, 1988.

RONDON NETO, R. M.; KOZERA, C.; ANDRADE, R. R. Caracterização florística estrutural de um fragmento de Floresta Ombrófila Mista, em Curitiba, PR - Brasil. Floresta, Curitiba, v. 32, n. 1, p. 3-16, 2002.

ROSEIRA, D. S. Composição florística fitossociológica do bosque com Araucaria angustifolia (Bertol.) Kuntze no Parque Estadual João Paulo II, Curitiba, Paraná. Dissertação (Mestrado em Botânica) - Setor de Ciências Biológicas, Universidade Federal do Paraná, Curitiba, 1990.

SANQUETTA, C. R. Dinâmica da Composição Florística de um fragmento de Floresta Ombrófila Mista no Centro-Sul do Paraná. Revista de Ciências Exatas e Naturais, Guarapuava, ano 1, n. 2, jan /junho, 2000.

SAINT-HILAIRE, A. Viagem à comarca de Curitiba (1820). São Paulo, Ed. Nacional, 1964.

SCHÄFFER, W. B.; PROCHNOW, M. A mata atlântica e você: como preservar, recuperar e se beneficiar da mais ameaçada floresta brasileira. Brasília, DF: Apremavi, 2002.

SILVA, F. C.; MARCONI, L. P. Fitossociologia de uma floresta com araucária em Colombo - PR. Boletim de Pesquisa Florestal, Colombo, n. 20, p. 23-28, 1990.

SOBRAL, M.; JARENKOW, J. A. Flora arbórea e arborescente do Rio Grande do Sul, Brasil. São Carlos: RiMa: Novo Ambiente, 2006.

STEVENS, P. F. Angiosperm Phylogeny Website. 2008. Versão 8, Junho de 2008. Disponível em: <http://www.mobot.org/MOBOT/research/APweb/>. Acesso em: 18/9/2009.

TABARELLI, M.; GASCON, C. Lições da pesquisa sobre fragmentação: aperfeiçoando políticas e diretrizes de manejo para conservação da biodiversidade. Megadiversidade, n. 1, v. 1, julho, 2005.

TABARELLI, M.; GASCON, C; SILVA, J. M. C. Forest fragmentation, synergisms and the impoverishment of neotropical forests. Biodiversity and Conservation, London, v. 13, p. 1419-1425, 2004.

TROPICOS. 2009 Tropicos.org. Missouri Botanical Garden. Disponível em: 〈http://www.tropicos.org>. Acesso em: 18/9/2009.

WORLD CHECKLIST OF SELECTED PLANT FAMILIES. The Board of Trustees of the Royal Botanic Gardens, Kew, 2008. Disponível em: <http://www.kew.org/wcsp/>. Acesso em: 18/9/2009. 